\title{
Alta Tecnologia e Efeito Placebo: o Caso da Revascularização Miocárdica a Laser no Tratamento Paliativo da Angina do Peito Refratária
}

High Technology and Placebo Effect: Myocardial Laser Revascularization in the Palliative Treatment of Refractory Angina Pectoris

Mário Sérgio Soares de Azeredo Coutinho

Universidade Federal de Santa Catarina, Instituto de Cardiologia de Santa Catarina - Florianópolis, SC

Desde 1933, quando Wearn e cols. ${ }^{1}$ descreveram uma rede de sinusóides no coração humano, algumas formas de revascularização indireta do miocárdio foram propostas na tentativa de melhorar sintomas cardio-isquêmicos. Em 1935, Beck $^{2}$ desenvolveu a cardio-omentopexia na qual o omento era suturado ao coração com o objetivo de aumentar sua perfusão. Em 1946, o cirurgião canadense Arthur Vineberg ${ }^{3}$ propôs o implante da artéria torácica interna diretamente sobre o miocárdio como forma de restabelecer o fluxo coronário em pacientes com angina. A ausência de resultados consistentes e o desenvolvimento das técnicas de revascularização coronária direta levaram ao progressivo abandono daquele procedimento. Em 1965, Sen e cols. ${ }^{4}$ propuseram a criação de canais transmurais na parede do ventrículo esquerdo para permitir a perfusão direta do miocárdio isquêmico com sangue oxigenado do VE. Este conceito se baseava no coração dos répteis, no qual o ventrículo esquerdo é diretamente perfundido por canais revestidos de endotélio que se irradiam da cavidade ventricular. Na década de 80, o conceito dos "sinusóides cardíacos" foi retomado e teve início a era da revascularização a laser ${ }^{5,6}$. Com o auxílio do Laser (Light Amplification by Stimulated Emission of Radiation) de dióxido de carbono ou de hólmio, o miocárdio de uma área supostamente isquêmica era perfurado com o objetivo de aumentar a perfusão e diminuir os sintomas na angina refratária. A abertura de canais com o uso do laser foi investigada histologicamente e não há evidência de que eles permaneçam pérvios após o procedimento; pelo contrário, eles são quase que imediatamente preenchidos com células e sangue e, assim, bloqueados. Outros mecanismos especulados para explicar a ação do laser na melhora da angina são a neoangiogênese e a denervação simpática cardíaca ${ }^{7}$, além do efeito placebo do procedimento. Os dois primeiros são também especulativos e não há prova definitiva de que eles estejam atuando no processo de melhora dos sintomas anginosos. O efeito placebo é outra possibilidade que será discutida adiante.

Atualmente há duas modalidades de revascularização a laser. A primeira é cirúrgica (TMLR-transmyocardial laser revascularization), feita através de uma pequena toracotomia lateral por onde são realizadas as perfurações com laser; a segunda é feita por via percutânea (PMLR-percutaneous myocardial laser revascularization). O cateter-laser é avançado até o ventrículo esquerdo e as perfurações são realizadas no sentido endocárdio-epicárdio. O primeiro método foi aprovado pelo FDA americano em 1998; o segundo é ainda considerado experimental. O FDA não aprovou o uso do laser concomitante à cirurgia de revascularização, embora, em um registro recente, $67 \%$ dos procedimentos tenham sido realizados em associação à cirurgia de revascularização do miocárdio ${ }^{8}$.

\section{Estudos randomizados duplos-cegos placebo- controlados}

Até o presente momento, foram publicados apenas 3 ensaios randomizados duplos-cego placebo-controlados com laser transmiocárdico, todos por via percutânea.

Stone e cols. ${ }^{9}$ avaliaram pacientes com angina refratária causada por uma ou mais oclusões totais de artérias coronárias nativas. Um total de 141 pacientes consecutivos com angina classes CCS (Canadian Cardiovascular Society) III-IV e com obstruções coronárias totais - nas quais a intervenção percutânea com balão falhou -, foram randomizados em 17 centros médicos para PMLR mais terapia medicamentosa máxima (TMM) ou só para TMM. O mascaramento ("cegamento") dos pacientes foi feito com sedação e uso de óculos escuros. A avaliação dos desfechos no seguimento foi feita sem o conhecimento da terapia empregada. Foram excluídos pacientes com FEVE $<30 \%$, IAM nos últimos 3 meses, impossibilidade de realização de teste de exercício, entre outros critérios. O desfecho primário foi a melhora no tempo de duração do teste ergométrico. A PMLR foi guiada por fluoroscopia e feita com laser de hólmio. O tempo de duração do teste ergométrico foi avaliado em apenas metade dos pacientes, 6 meses após o procedimento, e não mostrou diferença entre os grupos. Os sintomas anginosos após 6 meses também foram semelhantes entre os grupos. Não foram notadas diferenças nos eventos clínicos, incluindo mortalidade

\section{Palavras-chave}

Angina do peito refratária, revascularização a laser, efeito placebo. 


\section{Atualização Clínica}

e IAM, embora houvesse uma tendência maior de eventos no grupo laser (tamponamento, derrame pericárdico, taquicardiafibrilação ventricular, elevação de cardiomarcadores).

Salem e cols. ${ }^{10}$ randomizaram 82 pacientes com angina graus CCS III e IV para PMLR ou procedimento placebo. Os dois grupos eram comparáveis quanto às suas características basais e foram excluídos casos de infarto agudo do miocárdio, insuficiência cardíaca, angina instável requerendo hospitalização nos 14 dias que precederam o procedimento, entre outros. O desfecho primário foi a melhora da classe de angina (CCS). Na avaliação de 6 e 12 meses após o procedimento, houve melhora significativa do grupo tratado com PMLR quando comparado ao grupo placebo. Entretanto, nas medidas objetivas de tolerância ao exercício (tempo de esteira, taxa de troca respiratória e consumo de oxigênio) os dois grupos apresentaram resultados semelhantes. A fração de ejeção não se modificou após 12 meses de seguimento e a sobrevida livre de eventos foi semelhante nos 2 grupos ao final de 1 ano.

Leon e cols. ${ }^{11}$ realizaram ensaio randomizado duplocego e placebo-controlado com PMLR com 298 pacientes refratários à terapia usual com beta-bloqueador, antagonista do cálcio e nitrato. Eles foram randomizados para 3 grupos (laser placebo, dose baixa e dose alta de laser). O desfecho primário era a duração do teste ergométrico. Pacientes que receberam laser (dose baixa ou alta) tiveram mortalidade de 30 dias após o procedimento maior que o grupo placebo $(p=0,03)$. Aos seis meses, não houve diferença entre os 3 grupos na tolerância ao exercício. Da mesma forma, não houve melhora significativa de qualquer dos grupos quanto às classes CCS de angina. Também não houve mudanças na perfusão miocárdica medida pelo SPECT nuclear (repouso e stress) realizado 6 meses após o procedimento. Os autores concluem que "todos os outros estudos randomizados nessa área deveriam ser vistos com reservas e ceticismo, a não ser que atenção apropriada seja dada aos efeitos placebo das terapias experimentais a laser".

\section{Estudos randomizados abertos, sem grupo placebo, e comparados com terapia} medicamentosa

Existem 6 estudos randomizados controlados com terapia medicamentosa, sem grupos placebo e não-cegos publicados.

Frazier e cols. ${ }^{12}$ publicaram em 1999 os resultados de um estudo randomizado de 192 pacientes (91 tratados com TMLR e 101 com terapia medicamentosa), tendo como desfecho principal o grau de angina aferido pelo padrão CCS. Houve aferição aberta e cega que não mostraram diferenças entre si. Pacientes submetidos a TMLR tiveram escores de angina melhores quando comparados ao grupo de controle (67\% vs $20 \%)$. Não houve diferença na mortalidade de 12 meses entre os grupos. Da mesma forma, os estudos de perfusão SPECT com stress farmacológico não mostraram diferença entre os grupos em nenhum momento do seguimento, ou seja, não foi demonstrada evidência objetiva de melhora de perfusão com o procedimento. Dos 101 pacientes randomizados para terapia medicamentosa, 60 (59\%) acabaram sendo submetidos à TMLR, o que de alguma forma distorceu os resultados, já que a randomização foi desrespeitada. Os investigadores reconhecem essa fraqueza do estudo e argumentam que o cross-over para TMLR foi oferecido aos pacientes como um incentivo para permanecer no estudo. Isso mostra que houve um viés por parte dos investigadores, que tomaram uma atitude pró-TMLR. Diante dessas falhas, esse estudo foi duramente criticado no editorial que o acompanha ${ }^{13}$.

Allen e cols. ${ }^{14}$ (publicaram seus resultados simultaneamente ao estudo anterior. Foram randomizados 275 pacientes com angina refratária classe IV sem potencial para angioplastia ou cirurgia. Desses, 132 receberam TMLR e terapia medicamentosa, e 143 apenas terapia medicamentosa. Os parâmetros relacionados à classe da angina e tolerância ao exercício (esta, medida apenas em um terço dos indivíduos e não medida no início do estudo), assim como ao número de indivíduos considerados falhas terapêuticas foram melhores no grupo TMLR. A mortalidade não diferiu entre os grupos no seguimento de um ano. Entretanto, mais uma vez, os estudos de perfusão não mostraram diferença entre os grupos, colocando em cheque um dos mecanismos aventados para explicar os efeitos benéficos da terapia a laser. Também não se verificou melhora da função ventricular após o procedimento.

No editorial que acompanha os 2 estudos acima, Lange e Hills ${ }^{13}$ comentam que a TMLR não é isenta de riscos, e que nos estudos observacionais iniciais a mortalidade estava entre $10 \%$ e $20 \%$ e era maior nos indivíduos com fração de ejeção deprimida. Quando esses pacientes eram excluídos, a mortalidade reduzia-se a 5\%. A morbidade perioperatória se encontra, nos diversos estudos, entre 32\% e 68\% (infarto não fatal, insuficiência cardíaca, arritmias, e infecções cirúrgicas e respiratórias). Os editorialistas também comentam que os custos do procedimento não são desprezíveis e finalizam em um tom de ceticismo quanto ao procedimento de revascularização com laser.

Schofield e cols. ${ }^{15}$ estudaram 188 indivíduos com angina refratária no Reino Unido. Estes foram avaliados aos 3, 6 e 12 meses na sua capacidade física com teste em esteira e com o teste de 12 minutos de caminhada. Não foi constatada diferença nesses parâmetros entre os grupos randomizados para TMLR e terapia medicamentosa. A mortalidade foi similar entre os grupos, mas os escores para angina foram melhores no grupo TMLR. Mais uma vez, os estudos de perfusão não mostraram diferença entre os grupos após 12 meses, o mesmo acontecendo com a fração de ejeção do VE. Os editorialistas que comentam a contradição destes resultados com os de outro estudo, dizem que no estudo de March ${ }^{16}$ há inúmeros problemas na apresentação de dados importantes, que comprometem seriamente a interpretação dos achados. Finalizam dizendo que "relegar o laser apenas a um mero e caro procedimento analgésico arruína a maioria das expectativas daqueles que acreditavam em uma 'revascularização' pelo laser"17.

Burkhoff e cols ${ }^{18}$. foram os investigadores do estudo ATLANTIC (Angina Treatments-Lasers and Normal Therapies in Comparison). Eles recrutaram 182 pacientes de 16 centros nos EUA com angina III-IV CCS, isquemia reversível e resposta incompleta a outras terapias. Os pacientes foram 
randomizados para TMLR $(n=92)$ ou medicações $(n=90)$. Após 12 meses houve um aumento significativo na tolerância ao esforço no grupo TMLR, assim como nos escores de angina. Os estudos de perfusão e a fração de ejeção do VE não mostraram modificações significativas aos 3, 6 e 12 meses pós-procedimento em ambos os grupos.

O estudo PACIFIC (Potencial Angina Class Improvement From Transmyocardial Channels $)^{19}$ randomizou 221 pacientes classes III-IV com resposta precária à medicação para TMLR com laser de hólmio mais medicação, ou apenas medicação. O desfecho primário foi a tolerância ao exercício após 12 meses. Os resultados mostraram um aumento da tolerância ao exercício de 89 segundos comparado aos 12,5 segundos nos grupos TMLR e medicação $(p=0,008)$, respectivamente.

Os estudos ATLANTIC e PACIFIC foram inteiramente financiados pela indústria que produz o equipamento laser utilizado. Os investigadores reconhecem que um dos problemas com ambos os estudos foi o não-cegamento dos investigadores e pacientes, o que introduz um importante viés de aferição, principalmente quando se lida com desfechos subjetivos. Além disso, comentam que o mecanismo da eventual melhora dos pacientes é ainda desconhecido e não negam que o efeito placebo possa explicar, em parte, os resultados.

Um estudo norueguês conduzido por Aaberge e cols. ${ }^{20}$ randomizou 100 pacientes para terapia médica ou TMLR usando como desfecho primário os sintomas, a capacidade física e o consumo máximo de oxigênio aferidos pelo teste ergométrico. Embora os escores para angina tenham sido melhores no grupo TMLR em relação ao grupo tratado clinicamente, os parâmetros objetivos de capacidade física, como tempo de esteira e consumo máximo de oxigênio, não diferiram entre os grupos. A morbidade e mortalidade após 12 meses foi semelhante nos 2 grupos. Não se observou modificação na fração de ejeção após um ano do procedimento. Os autores comentam que o "tratamento cardíaco a laser" (refutando o termo 'revascularização transmiocárdica') pode funcionar na redução dos sintomas mas "não há evidência convincente de que ele melhore a função cardíaca ou reduza o número de eventos isquêmicos". Reconhecem que, para que se avalie o papel do "efeito placebo" sobre os resultados do procedimento são fundamentais os estudos duplos-cegos, placebo-controlados com PMLR.

Uma meta-análise de Liao e cols. ${ }^{21}$ incluiu 7 ensaios randomizados concluídos até 2003. Eram estudos randomizados abertos e sem controle com placebo. O grupo de controle era, usualmente, constituído por pacientes em uso da terapia convencional para angina. As conclusões da meta-análise são de que não há diferença na mortalidade entre os grupos com tratamento a laser e aqueles com tratamento-padrão. O grupo tratado com laser mostrou redução dos sintomas ( $\geq 2$ classes (CS) até um ano após o procedimento. Os autores não comentam sobre o papel dos estudos abertos e a ausência do controle-placebo como potenciais fontes de vieses.

\section{Estudos observacionais não controlados}

Estudos observacionais não são metodologicamente adequados para estudar a eficácia de intervenções na prática clínica. Em uma recente revisão sobre revascularização a laser no tratamento da coronariopatia refratária ${ }^{22}$, as várias séries de casos não controlados e não randomizados mostravam, de um modo geral, melhora sintomática e, em poucos estudos, melhora da capacidade física medida pelo tempo de exercício. Entretanto, não havia comprovação objetiva de melhora da perfusão miocárdica com os métodos de imagem utilizados. Como já é conhecido o fenômeno da maior presença de "estudos positivos" nos registros e séries de casos, essas conclusões não devem servir de norte para o uso de intervenções, mas apenas como geradoras de hipóteses a serem testadas em ensaios randomizados. Nesta revisão, os autores concluem que "os resultados dos recentes estudos duplos-cegos são conflitantes, sugerindo um efeito placebo significativo na resposta ao PMLR. Recomenda-se a realização de ensaios clínicos maiores para examinar o valor dessa técnica".

\section{Diretrizes}

A diretriz brasileira de doença coronariana crônica ${ }^{23}$ recomenda a TMLR como classe Ilb-B (aceitável, evidência razoável, considerado tratamento alternativo, baseado em pequenos estudos randomizados e estudos não randomizados e observacionais) reconhecendo que não há consenso quanto às suas indicações e a sua real eficácia. $\mathrm{O}$ estudo com 40 pacientes do Instituto do Coração, mencionado nas diretrizes, não aparece nas referências do documento. A única referência nacional é de uma série de 20 casos publicada em $1999^{24}$. A diretriz americana para angina estável ${ }^{25}$, publicada em 2002, recomenda a TMLR como classe Ila, mas reconhece que não existem estudos de longo prazo que avaliem os resultados dessa técnica. Também recomenda estudos para avaliar o desempenho da TMLR sozinha ou acompanhada de revascularização cirúrgica.

\section{Pesquisa científica, vieses e efeito placebo}

Toda pesquisa científica pode ser invalidada por erros sistemáticos chamados vieses. No caso do laser na angina refratária, as fontes de vieses começam pela avaliação da angina. Por se tratar de um sintoma, sua avaliação objetiva e precisa não é possível. Os 3 estudos randomizados ${ }^{9-11} \mathrm{e}$ cegos publicados até o momento não evidenciaram diferenças no tempo de exercício e na perfusão após o procedimento a laser quando comparados aos resultados do grupo de controle. Apenas um desses estudos ${ }^{10}$, com uma amostra pequena, evidenciou melhora dos sintomas 6 meses após o procedimento. Essas discrepâncias têm sido explicadas por diferenças técnicas na aplicação do laser ${ }^{26,27}$. Entretanto, é possível que as diferenças observadas entre estudos não-cegos e sem controle com placebo e aqueles randomizados, cegos e com placebo se deva a diferenças na metodologia do estudo mais que às questões técnicas do procedimento.

Em estudos clínicos, médicos e pacientes sempre têm expectativas quanto aos resultados do procedimento a ser avaliado. Pacientes altamente motivados e desesperados por uma nova forma de tratamento que alivie seus sintomas, naturalmente esperam por benefício clínico. A palavra "laser" está associada, no imaginário dos pacientes, e também no dos 
médicos, a uma tecnologia de ponta e de alta eficácia usada como "último recurso" ${ }^{28}$ no tratamento da angina. Portanto, há uma grande chance de que os sintomas sejam avaliados de forma distorcida, já que "se espera" que o laser produza um efeito positivo. Sabe-se, também que há uma relação direta entre o grau de stress e o efeito placebo, especialmente se o desfecho avaliado é a dor ${ }^{29}$.

O controle do viés de aferição é feito pelo duplo mascaramento (investigador e paciente) ou duplo-cego. O uso de placebo em estudos de intervenções novas é uma necessidade para definir sobre a sua utilidade ou futilidade. Até os mais recentes estudos com laser placebo, a terapia com laser na angina do peito era comparada com a terapia convencional medicamentosa. No caso da intervenção a laser transmiocárdica cirúrgica, o mascaramento era impraticável e os resultados eram, assim, enfraquecidos por vieses potenciais. No caso do laser percutâneo na angina, a comparação com placebo, embora metodologicamente mais complexa, é factível $^{9-11}$. No mais recente estudo randomizado que se publicou, comparando laser em duas "doses" e placebo laser" ${ }^{11}$, este grupo apresentou melhora de $30 \%$ nos sintomas e no tempo de exercício após o procedimento, resultado que não diferiu dos grupos que receberam o laser.

\section{Referências}

1. Wearn JT, Mettier SR, Klumpp TG, Zschiesche LJ. The nature of vascular communications between the coronary arteries and the chambers of the heart. Am Heart J. 1933; 9: 143-64.

2. Beck C. The development of a new blood supply to the heart by operation. Ann Surg. 1935; 102: 801-13.

3. Vineberg A. Development of anastomosis between coronary vessels and transplanted internal mammary artery. Can Med Assoc J. 1946; 55: 17-9.

4. Sen PK, Udwadia TE, Kinare SG, Parulkar GB. Transmyocardial acunpuncture: a new approach to myocardial revascularization. J Thorac Cardiovasc Surg. 1965; 50:181-9.

5. Mirhoseini M, Cayton MM. Revascularization of the heart by laser. J Microsurg. 1981; 2: 253-60.

6. Mirhoseini M, Muckerheide M, Cayton MM. Transventricular revascularization by laser. Lasers Surg Med. 1982; 2: 187-98.

7. Kim MC, Kini A, Sharma SK. Refractory angina pectoris: mechanism and therapeutic options. J Am Coll Cardiol. 2002; 39: 923-34.

8. Peterson ED, Kaul P, Kaczmarek RG, Hammill BG, Armstrong PW, Bridges CR, et al. From controlled trials to clinical practice: monitoring transmyocardial revascularization use and outcomes. J Am Coll Cardiol. 2003; 42: 1611-6.

9. Stone GW, Teirstein PS, Rubenstein R, Schmidt D, Whitlow PL, Kosinski EJ, et al. A prospective, multicenter, randomized trial of percutaneous transmyocardial laser revascularization in patients with nonrecanalizable chronic total occlusions. J Am Coll Cardiol. 2002; 39: 1581-7.

10. Salem M, Rotevatn S, Stavnes S, Brekke M, Vollset SE, Nordrehaug JE. Usefulness and safety of percutaneous myocardial laser revascularization for refractory angina pectoris. Am J Cardiol. 2004; 93: 1086-91.

11. Leon MB, Kornowski R, Downey WE, Weisz G, Baim DS, Bonow RO, et al. A blinded, randomized, placebo-controlled trial of percutaneous laser myocardial revascularization to improve angina symptoms in patients with severe coronary disease. J Am Coll Cardiol. 2005; 46: 1812-9.

12. Frazier OH, March RJ, Horvath KA. Transmyocardial revascularization with
O efeito placebo nas doenças cardiovasculares já tem sido descrito em várias situações, incluindo na angina ${ }^{30}$. Estima-se que a melhora dos sintomas em pacientes com angina crônica de esforço estável pode ser atingida, com placebo, em 30\% a $80 \%$ dos $\operatorname{casos}^{30}$. Procedimentos invasivos e cirúrgicos têm um grande potencial de efeito placebo ${ }^{31}$. O exemplo clássico de efeito placebo na cirurgia cardiovascular fica por conta da técnica de ligadura da artéria mamária interna no tratamento da angina. Esse procedimento foi proposto na década de 50 e usado rotineiramente até que um estudo randomizado ${ }^{32}$ usando um grupo de controle (fazia-se a incisão torácica sem ligar a artéria) mostrou que não havia diferença entre os grupos quanto à melhora dos sintomas. Ou seja, o mero ato de seccionar a pele do tórax foi capaz de mostrar benefícios subjetivos.

Nesta revisão procuramos sintetizar a experiência clínicocientífica do uso da terapia a laser no tratamento da angina do peito refratária. Estudos observacionais e randomizados preliminares mostravam resultados positivos desta terapia. Entretanto, estudos randomizados e placebo-controlados e, portanto, metodologicamente mais robustos, apontam fortemente para a possibilidade da existência de efeito placebo da terapia a laser na angina refratária. a carbon dioxide laser in patients with end-stage coronary artery disease. $\mathrm{N}$ Engl J Med. 1999; 341: 1021-8.

13. Lange RA, Hillis LD. Transmyocardial laser revascularization. N Engl J Med. 1999; 341: 1075-6.

14. Allen KB, Dowling RD, Fudge TL, Schoettle GP, Selinger SL, Gangahar DM, et al. Comparison of transmyocardial revascularization with medical therapy in patients with refractory angina. N Engl J Med. 1999; 341: 1029-36.

15. Schofield PM, Sharples LD, Caine N, Burns S, Tait S, Wistow T, et al. Transmyocardial laser revascularization in patients with refractory angina: a randomised controlled trial. Lancet. 1999; 353: 519-24.

16. March RJ. Transmyocardial laser revascularization with the $\mathrm{CO} 2$ laser: one year results of a randomized, controlled trial. Semin Thorac Cardiovasc Surg. 1999; 11: 12-8.

17. Pretre R, Turina MI. Laser to the heart: magic but costly, or only costly? Lancet. 1999; 353: 512-3.

18. Burkhoff D, Schmidt S, Schulman SP, Myers J, Resar J, Becker LC, et al. Transmyocardial laser revascularization compared with continued medical therapy for treatment of refractory angina pectoris: a prospective randomised trial. ATLANTIC Investigators. Angina Treatments-Lasers and Normal Therapies in Comparison. Lancet. 1999; 354: 885-90.

19. Oesterle SN, Sanborn TA, Ali N, Resar J, Ramee SR, Heuser R, et al. Percutaneous transmyocardial laser revascularization for severe angina: the PACIFIC randomised trial. Potential Class Improvement From Intramyocardial Channels. Lancet. 2000; 356: 1705-10.

20. Aaberge L, Nordstrand K, Dragsund M, Saatvedt K, Endresen K, Golf S, et al. Transmyocardial revascularization with $\mathrm{CO} 2$ laser in patients with refractory angina pectoris. Clinical results from the Norwegian randomized trial. J Am Coll Cardiol. 2000; 35: 1170-7.

21. Liao L, Sarria-Santamera A, Matchar DB, Huntington A, Lin S, Whellan DJ, et al. Meta-analysis of survival and relief of angina pectoris after transmyocardial revascularization. Am J Cardiol. 2005; 95: 1243-5.

22. Saririan M, Eisenberg MJ. Myocardial laser revascularization for the treatment 
of end-stage coronary artery disease. J Am Coll Cardiol. 2003; 41: 173-83.

23. Diretrizes de doença coronariana crônica: angina estável. Arq Bras Cardiol. 2004; 83 (Supl 2): 37-8.

24. Oliveira SA, Dallan LA, Lisboa LA, Chavantes MC, César LA, Pardi MJ, et al. Revascularização transmiocárdica com laser de CO2: experiência clínica inicial. Arq Bras Cardiol. 1999; 72: 441- 50.

25. Modest GA. Guidelines for the management of patients with chronic stable angina. Ann Intern Med. 2002; 137: 548-9; author reply 548-9.

26. Mouli SK, Fronza J, Greene R, Robert ES, Horvath KA. What is the optimal channel density for transmyocardial laser revascularization? Ann Thorac Surg. 2004; 78:1326-31; discussion 1326-31.

27. Hamawy AH, Lee LY, Samy SA, Polce DR, Szulc M, Vazquez M, et al. Transmyocardial laser revascularization dose response: enhanced perfusion in a porcine ischemia model as a function of channel density. Ann Thorac Surg. $2001 ; 72: 817-22$.

28. Kaptchuk TJ, Goldman P, Stone DA, Stason WB. Do medical devices have enhanced placebo effects? J Clin Epidemiol. 2000; 53: 786-92.

29. Hrobjartsson A, Gotzsche PC. Is the placebo powerless? An analysis of clinical trials comparing placebo with no treatment. N Engl J Med. 2001; 344: 1594 602.

30. Bienenfeld L, Frishman W, Glasser SP. The placebo effect in cardiovascular disease. Am Heart J. 1996; 132: 1207-21.

31. Johnson AG. Surgery as a placebo. Lancet 1994; 344: 1140-2.

32. Dimond EG, Kittle CF, CrockettJE. Comparison of internal mammary artery ligation and sham operation for angina pectoris. Am J Cardiol. 1960; 5: 483-6. 